\title{
The Role of Tumor Banking and Related Informatics
}

\author{
Stephen J. Qualman, Jay Bowen, \\ Sandra Brewer-Swartz, and Mary France
}

\section{INTRODUCTION}

The majority of this book deals with the diagnostic and research applications of molecular or tissue array technologies with regard to expression profiling of tumors. These efforts (1) will only be successful with the logical application of tumor banking and its associated informatics systems as the translational bridge linking new molecular information to its clinical significance. The design of tumor banks should be such that significant effort is devoted to obtaining data on clinical outcomes (2), which permits investigators to know that such data are available for analysis as they pursue their molecular studies on bank-derived specimens. Tumor banking and its associated inventory informatics have been recognized for over a decade $(3,4)$ as necessary tools to advance the science of molecular testing; however, it has only been in the last 2 to $3 \mathrm{yr}$ that the linkage to clinical outcomes has been seen as crucial to achieving this goal (3). It is estimated that by the year 2005 (5), as much as $10 \%$ of clinical laboratory tests will be based on RNA or DNA analysis.

This chapter will deal with the broad concepts of tumor banking and informatics as practiced at the Biopathology Centers (BPC) located at the Children's Research Institute, Columbus, $\mathrm{OH}$. The BPC provides banking and informatics services to a variety of cooperative groups, including the pediatric Cooperative Human Tissue Network (pCHTN), Children's Oncology Group (COG), Gynecologic Oncology Group (GOG), and Childhood Cancer Survivor Study (CCSS). The BPC deals with patient specimens that span the age range of newborns to the elderly; procuring tumor types that include the spectrum of pediatric solid tumors, selected adult gynecologic tumors (ovarian, cervical, uterine), and any second malignant (solid) neoplasm in childhood cancer survivors. In the last decade, the BPC has served nearly 85,000 tumor or related tissue specimens to over 300 different investigators. These specimens are linked to patient clinical outcome, because of the BPC's alliance with the aforementioned cooperative groups.

This chapter will describe the BPC's structure with regard to its physical repository and related tumor-tissue procurement activities, as well as its informatics system as it relates to data elements, security and data encryption, and specimen inventory. Finally,

From: Expression Profiling of Human Tumors: Diagnostic and Research Applications

Edited by: Marc Ladanyi and William L. Gerald @ Humana Press Inc., Totowa, NJ 
the metrics, which are used to assess both the BPC's quality control activities and the BPC's impact on advancing medical science, will be discussed. It is hoped that documentation of the BPC's experiences will serve as a paradigm for others who plan to procure, bank, and utilize human tumor tissues for research purposes, such as expression profiling.

\section{BIOREPOSITORY STRUCTURE}

\section{Tissue Sources}

\section{Cooperative Group Affiliations}

The types of specimens that are procured, processed, and stored by the BPC are dependent upon what is needed by the cooperative groups. By collecting every type of specimen associated with each group's protocols, the BPC can assure each group of uniformity in storage and quality control. While snap-frozen tumor tissue and paraffin blocks comprise the majority of preparations, the BPC stores everything from buccal cells to urine.

Cooperative group affiliations have been beneficial to the BPC, because they have allowed the BPC to be involved in protocol development, performance monitoring, and educating institutional members. The groups have benefited from utilizing the BPC banking and pathology services as well, because the BPC provides shipping containers, specimen procurement supplies, use of a courier account number, and customer service to the institutions. In addition, the groups know that specimens linked to their protocols are being managed by experienced staff who stay abreast of the ever changing shipping and patient protection regulations.

\section{Local Institution(s)}

The BPC is located on the Columbus Children's Hospital campus and maintains a relationship with the Pathology Department in order to access surgical and autopsy specimens processed by that department. It is the responsibility of the BPC to inform the surgical pathologists as to what cases and specimens are of interest to the bank researchers. Laminated wall charts are provided for posting in the surgical suite, the frozen section room, the histology laboratory, or the autopsy suite, which illustrate the types of tissue that can be procured according to tumor type (Fig. 1). These are also distributed to outside BPC procurement sites. Proof of consent to use the tissue for research must be obtained before the tissue is stored in the BPC repository.

\section{Funding Sources}

In addition to the pCHTN funding for tumor procurement, the BPC is supported by the GOG, COG and CCSS in order to provide these groups with banking services and pathology services. All funding is either direct or derivative funding from the National Institutes of Health (NIH). The baseline amount of funding required to establish a minimal tissue procurement service, independent of personnel costs, is estimated to be around $\$ 20,000$ (2). This cost is also exclusive of space (4) and can be further broken down into initial equipment costs in freezing-storage apparatus, database software, and annual consumable costs (4). 


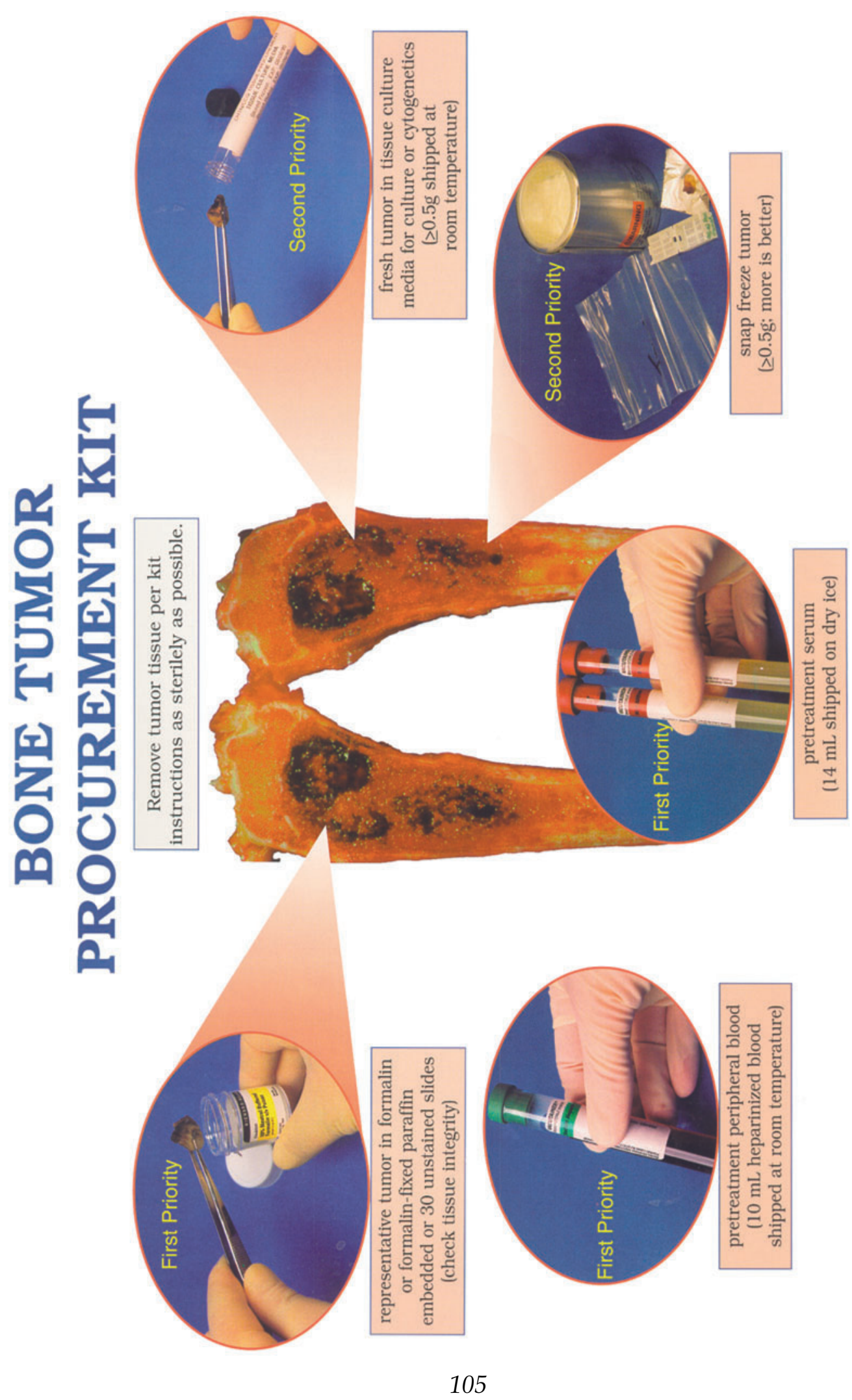

్ㅡㄹ

胥

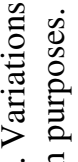

.

क.

कै प्र

芩

를

ö.

총

풍 믕

증

정크

응 롱

के

寻

I

I

ㅇํㅇ

들

드웜

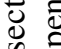

क वे

ํํㄹ

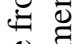

흔

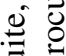

के

조

क.

के

\&

$\Xi \lesssim$

bo

记

ชิ

के

볼

突告

긍

3

- 월

ติ

赔 


\section{Space}

The BPC presently occupies $2400 \mathrm{ft}^{2}$ of laboratory, storage, and office space. Approximately $640 \mathrm{ft}^{2}$ is used as laboratory space for histology and processing and distribution of specimens. With sufficient space, specimen processing and distribution can be done in the same laboratory.

Various types of storage space are needed by the BPC, including $300 \mathrm{ft}^{2}$ for liquid nitrogen vapor-phase freezers, $-80^{\circ} \mathrm{C}$ freezers, and a cold room $\left(4^{\circ} \mathrm{C}\right)$, while $150 \mathrm{ft}^{2}$ is needed for storage of slides, paraffin blocks, touch preparations, and procurement kits. A minimum of $150 \mathrm{ft}^{2}$ is estimated to be required for repository purposes (2). Paperwork, which needs to be accessible to staff, is stored in filing systems within the office space of the BPC. Several rooms are utilized as office space for the BPC staff, totaling $1300 \mathrm{ft}^{2}$. Materials that are not frequently accessed may be stored off-site.

\section{Equipment and Staff}

Some of the pieces of equipment mentioned below are optional items, but were deemed as necessary due to the high activity level of the BPC. Laboratory-related staff consists of two histology technicians, two medical laboratory technicians, two research assistants, and one research coordinator. A minimum of two employees are probably required (4) to operate a biorepository, besides a pathologist to provide oversight; these include a bank coordinator, who maintains the tissue archive in all its aspects, and a histotechnician, to process tissues for distribution to investigators.

\section{Processing and Distribution Laboratories}

The specimens to be banked and/or served to bank researchers are received in the processing laboratory where they are weighed, labeled, and when necessary, further processed before being stored or served. The following equipment is routinely used by the processing and distribution laboratory staff: balances (precision to $0.01 \mathrm{~g}$ ), centrifuge (Ficoll-Hypaque isolation of white blood cell pellets), chemical fume hood, laminar flow hood (sterile handling), and a refrigerator. To maintain tissue integrity, a heat sealer is used for sealing specimens in plastic bags prior to storage at $-80^{\circ} \mathrm{C}$ and a Super Mutt Vacuum Sealer (Gramatech, Montebello, CA, USA) is used to package formalin-fixed tissues (paraffin blocks, scrolls, and slides) in mylar bags.

\section{Storage Facility}

Most tissues at the BPC are stored in liquid nitrogen vapor-phase freezers, because specimens kept at approximately $-170^{\circ} \mathrm{C}$ are of higher integrity than those maintained at higher temperatures and show less desiccation $(2,6)$. Freezers $\left(-80^{\circ} \mathrm{C}\right)$ are used for short-term tissue storage, and $-20^{\circ} \mathrm{C}$ freezers are used for reagent storage. Manual defrost units are preferred, as the freeze-thaw cycles of automatic defrost units can degrade the quality of biologics and reagents.

Paraffin blocks, scrolls, and slides are best preserved in vacuum-sealed mylar bags with a commercial oxygen absorber at $4^{\circ} \mathrm{C}$. Paraffin slides may also be dipped in molten paraffin prior to storage to preserve tissue antigenicity (7). The BPC utilizes a cold room (walk-in cooler) $\left(4^{\circ} \mathrm{C}\right)$ for storing its vacuum-sealed unstained slides and paraffin blocks. 
Touch preparations, taken and fixed from fresh tumor specimens, were once standard submissions to the BPC, but are now best performed from segments of snapfrozen tissue stored at the bank. These can then be fixed and sent to the investigator with relatively good preservation of antigenicity and morphology.

\section{Histology Laboratory}

The histology technicians process fixed specimens and provide review materials to be used in diagnosing the tissues of the BPC. This includes processing formalin-fixed tissue into paraffin blocks and preparing an hematoxylin and eosin (H\&E) section from each paraffin block for quality control review, as well as preparing fixed tissues for investigators (H\&E sections, tissue scrolls for ploidy studies, immunoperoxidase slides, etc.). Frozen sections from optimal cutting temperature (OCT)-embedded frozen tissues are also routinely prepared for immunohistochemistry, in situ hybridizations, or nucleic acid extractions. The equipment located in the BPC histology laboratory includes a tissue microarray instrument, a microtome, a cryostat, a tissue processor to prepare tissue for embedding, an embedding center, and a slide-labeling machine (the latter labels all slides using the BPC's anonymous numbering system in order to protect patient confidentiality).

\section{Specimen Procurement Kits and Storage}

The BPC provides submitting institutions with specimen procurement kits to facilitate the procurement process and encourage specimen submission. The specimen procurement kits are reusable, insulated, multitemperature specimen containers (Insulated Shipping Containers, Phoenix, AZ, USA), which allow shipment of both frozen and ambient temperature specimens in the same package (see Fig. 2A). This kit maintains ambient and frozen temperatures for more than $48 \mathrm{~h}$, despite the external environment through which it is transported (Fig. 2B).

The contents of kits are based on the particular group studies. Specimen procurement kits can include all the following kit supplies: OCT molds, $15-\mathrm{mL}$ formalin jars in styrofoam mailers, foil and specimen baggies, $1.5-\mathrm{mL}$ vials containing $1 \mathrm{~mL}$ of RNAlater ${ }^{\mathrm{TM}}$ (Ambion, Austin, TX, USA) for tissue, 2-mL tubes for serum, 2-mL tubes for urine, 5-mL ethylenediaminetetraacetic acid (EDTA) tubes for anti-coagulated blood or bone marrow, $15-\mathrm{mL}$ culture tubes, parafilm, charged glass slides in slide mailers, biohazard stickers, and dry ice labels.

Within the BPC, RNA is extracted from cryostat sections and tissues received in RNAlater. Some nucleic acid preservation products may reduce the usefulness of specimens for proteomics, so this consideration should be taken into account in the design of tissue protocols. Tissues sent to investigators for RNA extraction by the BPC may utilize RNAlater and obviate use of dry ice.

Long-term storage of specimens in vapor-phase liquid nitrogen freezers is done in small segments (e.g., $5 \mathrm{~g}$ or less) in plastic histology cassettes or mega-cassettes, with the specimens wrapped in aluminum foil to minimize desiccation. OCT-embedded specimens in cryomolds should likewise be wrapped in foil. Glass vials and pop-top plastic vials are not adequate storage containers for vapor-phase liquid nitrogen temperatures, as they may readily break or pop open (2). Screw-cap cryovials work well for storage of serum or urine. 


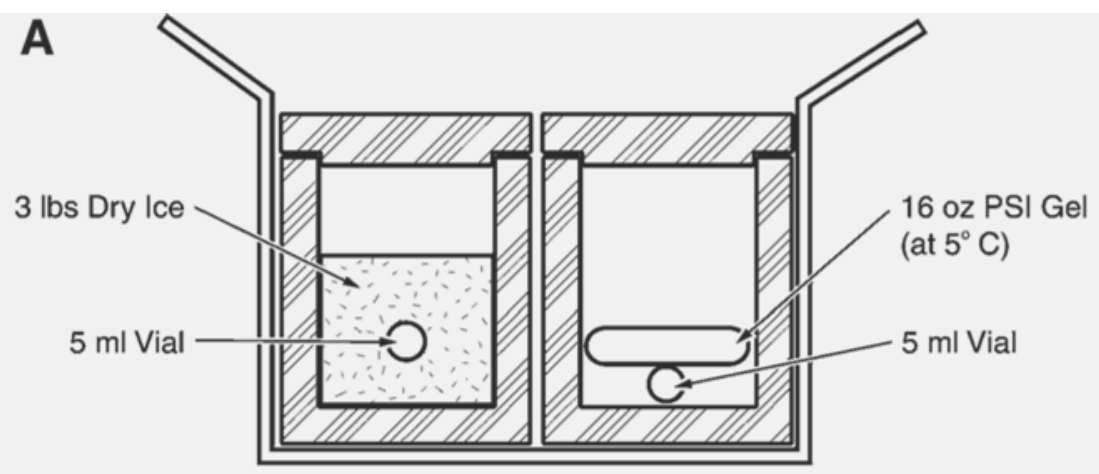

E-11C Dual Shipper

(Side View)

Manufactured by Insulated Shipping Containers, Inc., Phoenix, AZ

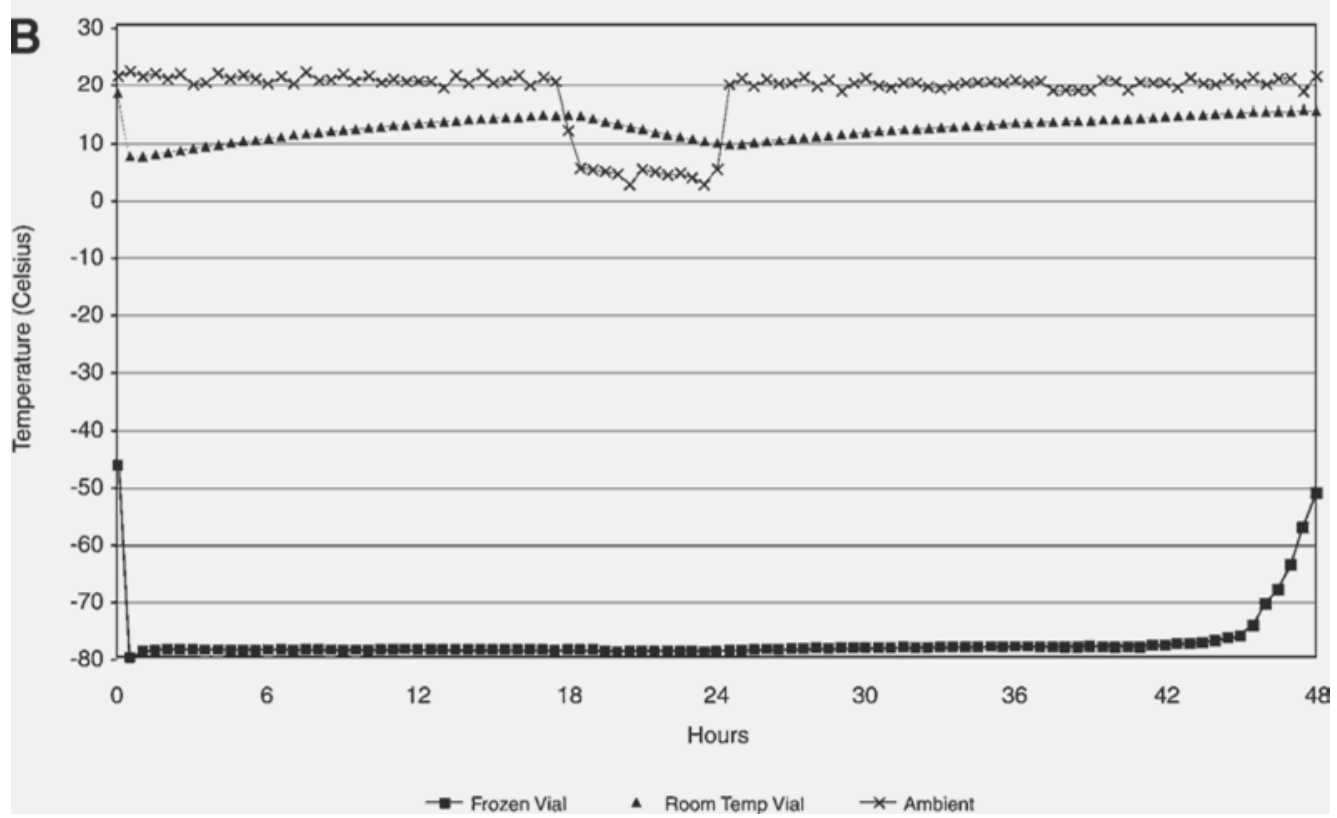

Fig. 2. (A) Insulated dual-chamber shipping container. One chamber contains dry ice for frozen specimens, while the other maintains specimens at room temperature using a gel pack. (B) Field testing of the kit shows dual maintenance of frozen and room temperature with associated specimens in the kit chambers (despite variation in outside ambient temperature) for a 48-h period.

\section{Specimen Transport}

Cooperative group-affiliated institutions must send specimens to the BPC through air-express couriers within $24 \mathrm{~h}$ of procurement, using the dual-chamber (Fig. 2) kits previously described. Shipments made via overnight carrier must be packaged to conform with International Air Transport Association (IATA) Section 1.5.0.2 and Code of Federal Regulations Section 172.7 (details available from the Web sites of various 
express courier companies and biohazardous shipping container companies). Researchers receiving specimens are billed for shipping charges and an additional specimenprocessing fee associated with the types and numbers of specimens served.

\section{DATA MANAGEMENT AND INFORMATICS}

\section{Data Management}

The BPC staffs includes three informatics-related employees (computer engineer, programmer, and database liaison). The computer engineer is on staff during system design and redesign and is only cost-effective as a temporary position. The programmer is needed full-time, because systems are always being amended to meet the changing needs of the groups. The database liaison works with the staff in assuring that the systems are meeting the needs of the users (defining them for the programmer) and is also responsible for sharing data with cooperative groups. The majority of documents maintained by the BPC are registration documents, operative reports, and pathology reports. Copies of the associated pathology reports are sent along to researchers with submitted specimens, but are stripped of patient identifiers. To protect patient confidentiality, documents with patient identifiers are currently kept in lockable cabinets in locked rooms.

\section{Informatics}

Informatics can facilitate patient registration, specimen tracking, tissue cataloging, quality assurance, and specimen availability. The ability of databases to organize and present desired information can also aid in tracking informed consent and institutional compliance and be used to generate tissue bank inventory reports to match investigator requests with specimen availability.

\section{Design Objectives for a Banking Inventory System}

The components of a system must appear seamless to allow for efficient data entry, queries and report preparation, and must also allow for rapid deployment of new services. Consideration should also be made for the future, as systems will become increasingly diverse; supporting multiple architectures, platforms, and databases. Exchange of information between different databases at different institutions may also be or become a concern.

Ideally, an informatics system ensures that data is available over a long period of time, maintained in a standardized format, able to be disseminated to others as needed, and collected from collaborative sources and combined.

\section{Informatics Programming Standards and Common Data Elements}

Information Services departments may have database technology standards from which a database system can be based, but often there are none. Children's Research Institute Core Technologies (CRICT) are the standards used by Children's Research Institute in Columbus, $\mathrm{OH}$, and were used as the starting point for the BPC's informatics system. These standards were then customized to meet the specific requirements of the project, resulting in a more specific set of standards called the BIOPATH II Database Architecture Guide (8). These standards allowed $80 \%$ of all banking and pathology review systems to be part of the core data structure, leaving only $20 \%$ for customized programming. 
The Cooperative Group Chairs, along with the National Cancer Institute, recognized the need for common data elements across data systems. As a result, the Intergroup Specimen Banking Committee was formed in 1999 to "identify existing standards, policies, and procedures, particularly those in common usage, which would address the needs of intergroup banking, rather than invent new ones. The goal was to deliver a set of recommendations that would require the least amount of work, change, and expense to implement, while providing sufficient guidance to the research community to facilitate useful banking efforts in support of correlative science, to avoid unnecessary conflict, and to ensure a sufficient standard of quality." (9).

Rather than establish standards of hardware, operating systems, or applications, the report instead set forth a standard for mandatory common data elements. These common elements provide for such things as uniform reporting, data exchange, and joint analysis.

Entities subject to these guidelines are medical institutions that submit specimens to one or multiple tissue banks-repositories, science review committees, investigators receiving specimens and responsible for submitting results, and statistical centers (9).

Minimum Data Requirements For Banking (9)

- All samples and subsamples must have a unique identifier.

- The system must adhere to standard coding mechanism.

- The system must be able to maintain an up-to-date inventory by a unique identifier to include the original and current quantity of specimens and where they have been sent, when (if) they have been returned, and when they have been exhausted. The system must also be able to invalidate samples.

- The database containing information on sample locations (inventory) may reside at the bank and does not need to be accessible by systems outside the bank.

- The system must be able to flag (reserve) specimens for a particular study.

- The system must track the type of informed consent for research that is allowed.

- The system must track specimens and allow for withdrawal of consent.

- The system must be able to report and provide query results across groups to facilitate any combination of a bank per trial, a bank per disease site, or a bank per group per disease site. Projects may require samples accrued on more than one trial.

- The system must provide quality control-checks on imported data.

\section{INFORMEd CONSENT AND CONFIDENTIALITY}

Tracking and cataloging informed consent is the key to banking informatics. Informed consent is the factor by which all tissues are qualified or disqualified for use by a potential researcher. The Intergroup Specimen Banking Committee recently endorsed the use of a three-item checkbox format for summarizing levels of informed consent (9). These levels allow for the patient to designate whether their tissue or case data may be used for: (i) cancer research; (ii) general medical research; and (iii) future patient contact for needed clinical follow-up.

\section{LiNKAGE AND De-LiNKAgE}

Risks posed to subjects from research with their tissues are strongly related to the identifiability of individual sources of those tissues (10). Data records, which can be directly or indirectly associated with a person's name or other identifying information, are referred to as linked data. Such identifying elements are date of birth, treating institution, treating physician, medical record numbers, social security numbers, etc. 


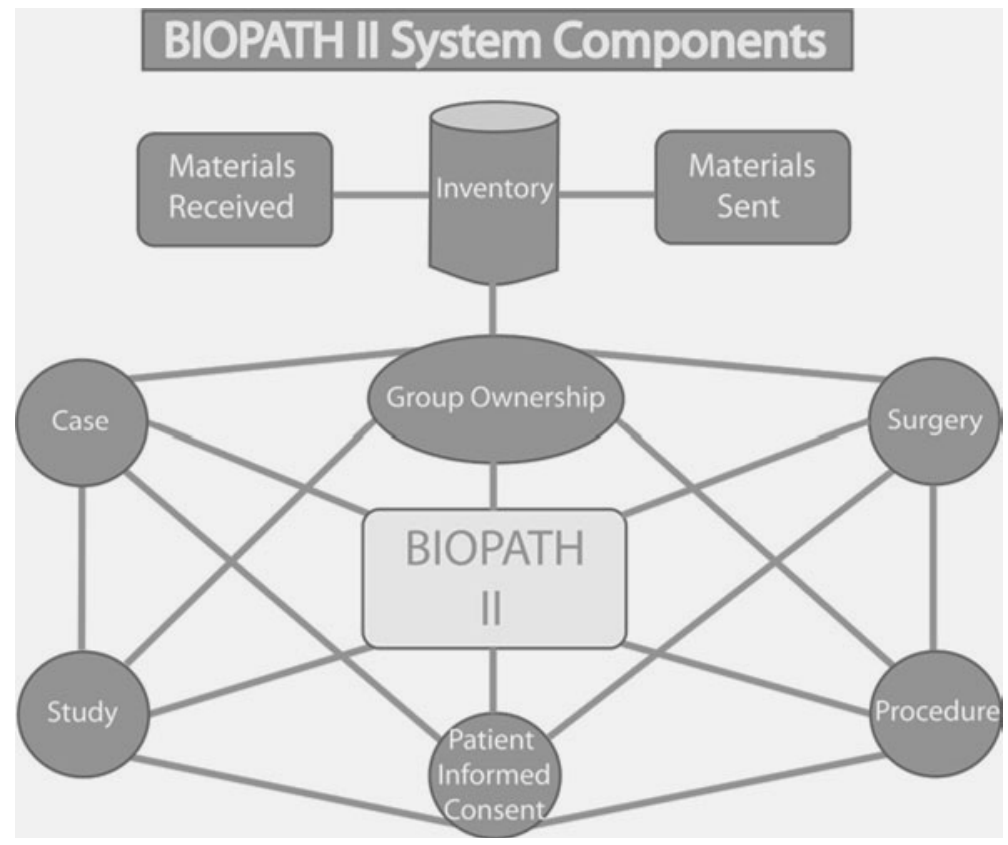

Fig. 3. Informatics design of BIOPATH II. A centralized inventory system is at the heart of the design, which can account for both cooperative group and patient-specific data, while maintaining a real-time inventory of specimen deposits, withdrawals, and residual tissues.

The BIOPATH II system uses specialized codes that, by default, "de-link" the specimen and clinical outcome data to maintain patient anonymity. If there is patient consent for the data to be linked, the data sets at the BPC and at a Cooperative Group Statistical Data Center are connected via a virtual private network (see section entitled Virtual Private Networks), and decoding can occur.

To permanently de-link data, any codes that will link or identify data or tissues with the donor must be removed, such that even the database manager can no longer trace a tissue or its related data back to the donor.

\section{Centralized Inventory System}

Inventory is at the heart of the tissue bank informatics design (Fig. 3). The inventory module is linked to cataloging, which provides for tagging and tracking of such things as informed consent, group ownership, and tissues reserved or specifically collected for a protocol or study. This ensures that specimens are tracked and distributed appropriately and in compliance with the patient's informed consent, as well as preventing the total depletion of a tissue sample.

The system design also streamlines tissue intake and serving of investigators. Materials are received and processed by a research assistant and entered directly into the system, while specimen shipments are processed through the same interface using the shipment module. The modules keep a precise record of materials as they are received and shipped, to produce a real-time inventory of what is available. When specimens are received and inventoried into the central system, the specimen is linked back to the specific cooperative group's case file. A full history of the specimen, from when it was 
received to when it was served, is maintained per cooperative group per case. Such history gives quick and easy access for reporting purposes.

\section{Virtual Private Networks}

A virtual private network (VPN) allows for usage of the Internet, normally a nonsecure medium, for transmission of confidential patient and specimen information using data encryption.

The use of virtual private networking has been fundamental in streamlining the patient enrollment and specimen registration process, reducing repetitive keying of data, and ensuring that information is synchronized between physically separate sites. This linking of databases can also be used to create virtual tissue banks from the inventory of different sites, thus allowing for the optimal matching of requests and distribution of tissues to reach a larger market.

The sensitive nature of medical information makes unauthorized disclosures and data alterations a concern. VPNs can be a cost-effective solution to provide secure point-to-point transactions utilizing encryption technologies. The primary requirements for implementation of a secure VPN are:

- Authentication; each end point checks the other and verifies that the transaction belongs to the secure point-to-point site before accepting the transaction or request.

- Strong data encryption to protect sensitive information.

- Transaction privacy (public-private key encryption).

- Encryption using a key unique to each information exchange session.

- Scalability; a VPN needs to be able to grow to accommodate an increase in connected sites.

- Ability to provide audit information.

- Immediate intrusion-attack detection and response; requires continuous evaluation of security policies and practices.

\section{METRICS OF PERFORMANCE}

\section{Areas of Assessment}

Creation of a physical biorepository, receipt of tissues, and development of an informatics system to monitor all aspects of specimen procurement and dispersal are merely a starting point for a successful tumor banking operation. Metrics or measures of the success of a banking operation are best assessed by looking at output, including what is served, who is served, and what is published. Metrics of what is served can include evaluations that are both morphologic and molecular in nature. This is in addition to written evaluations received from investigators served.

\section{Morphologic Assessment}

Central morphologic reviews are performed on the formalin-fixed tissues sent via the dual-chamber kits, which represent the mirror-image of samples of snap-frozen tumor and normal adjacent tissue also included in the kit. The histologic analyses are encoded on an informatics form for each histologic type of tumor examined and include such parameters as assessment of tumor diagnosis, percent tumor, percent stroma, and percent necrosis.

In a BPC sample of 7000 pediatric and gynecologic tumors examined over a $5-y r$ period, a diagnostic discrepancy rate of approx $10 \%$, between central and institutional diagnoses, was identified. Although this discrepancy rate might at first appear to 


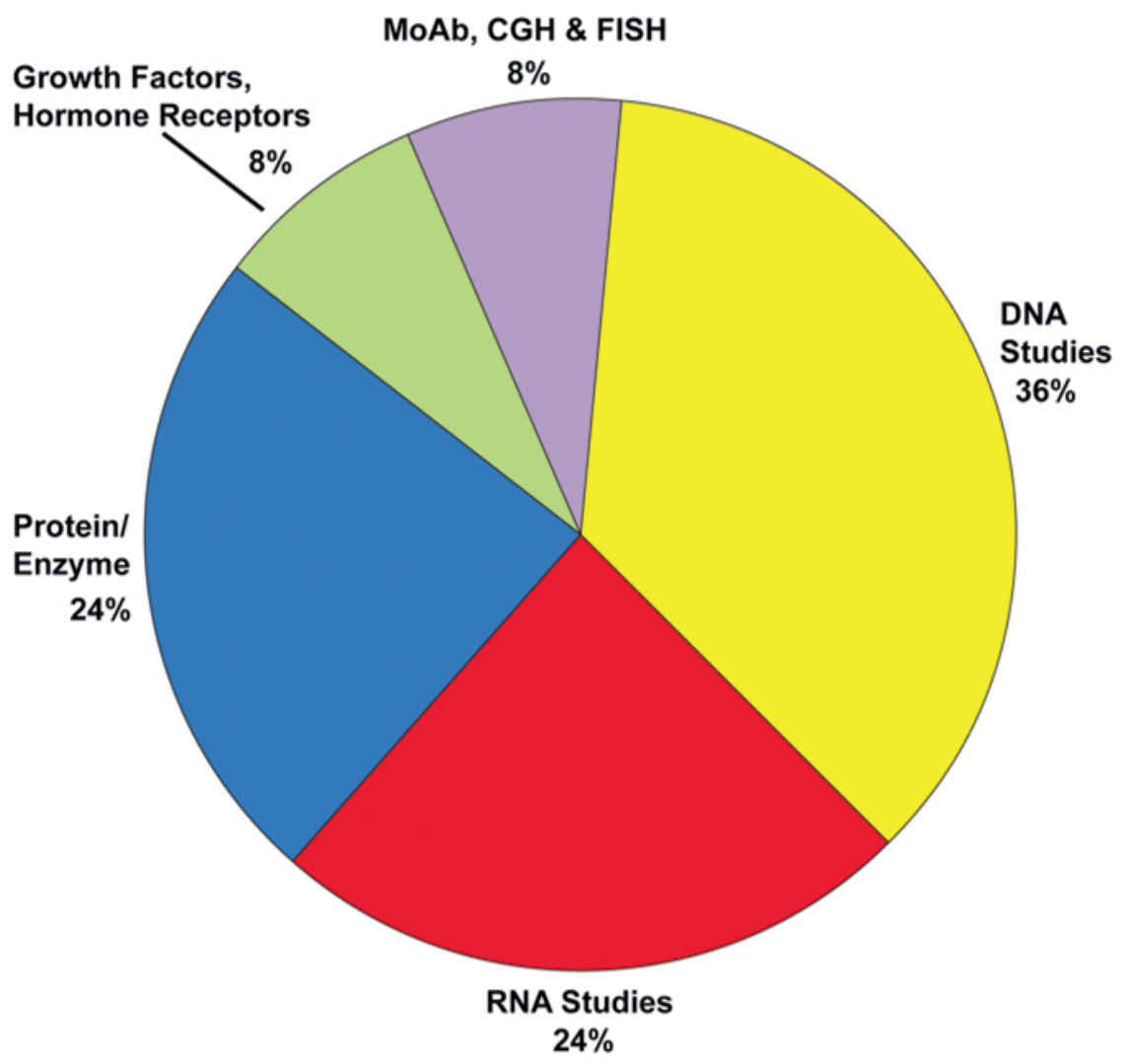

Fig. 4. Investigator uses of bank tissues as determined by direct query on feedback questionnaires. Sixty-percent (60\%) of tissues are used for nucleic acid studies, nearly $25 \%$ for protein-based studies, and the rest for hybridizations (CGH and FISH) along with immunohistochemistry. MoAb, monoclonal antibody.

be quite high, these data in the main reflect sampling problems identified in tissue aliquots labeled by the institution as "normal" or "tumor," which were not reflective of the aliquot's content (e.g., tissue totally necrotic, normal tissue contaminated by tumor, etc.). Less than $2 \%$ of cases contained true discrepancies between central review and institutional diagnoses (most of which were minor, reflecting differing opinions as to tumor grade or subtype). The $10 \%$ discrepancy rate does emphasize the need for morphologic review of submitted tissues, so that extremely necrotic or contaminated tissue aliquots ("normal" with tumor) are not used by investigators. The central review diagnosis is the one reported to investigators.

\section{Molecular Assessment}

Confirmation of morphologic viability of tissues is only the first step in assessing the quality of tissues served to investigators. A survey of our pediatric and gynecologic investigators, as to uses of tissues for research (Fig. 4), revealed that the preponderance of specimens are used for various types of molecular studies (60\% for RNA or DNA studies), with nearly one-third utilized for protein-based studies (including enzymes, growth factors, and hormone receptors). Studies involving hybridizations (fluorescence 
in situ hybridization [FISH], comparative genome hybridization [CGH]) or immunohistochemistry are in the minority (8\% of total studies).

With such emphasis on molecular uses of BPC tissues, efforts to assess the molecular integrity of tissue became necessary. The BPC has looked at the molecular integrity of its gynecologic specimens by reverse transcription polymerase chain reaction (RT-PCR) and DNA and RNA electrophoresis performed on ovarian tissues (11). RT-PCR assay for amplification of the mRNA gene product (177-bp product) of the HPRT housekeeping gene revealed adequate amplification in $70 \%$ of ovarian cases. RNA electrophoresis (followed by visual estimate of the $18 \mathrm{~s}$ and $28 \mathrm{~s}$ ribosomal RNA bands by ethidium bromide staining) also revealed RNA to be of good quality with minimal degradation in $70 \%$ of ovarian tissues. DNA electrophoresis showed the genomic DNA was of good quality in $100 \%$ of ovarian tissues tested. The challenge of adequate mRNA preservation is further discussed in the section entitled "Future Challenges."

Investigator Profiles and Publications

Another metric used to assess the effectiveness of a banking operation is defining who is served and what is published. These questions are answered during the feedback questionnaire process by asking for updated investigator biosketches and project summaries, along with a summary of current and submitted grant proposals (including those based on uses of BPC tissues). Direct queries of the investigators are also made on an annual basis regarding any publications they have produced based on BPC tissues, in part or total, and this list is compared with Internet listings of the same. All authors are asked to acknowledge BPC contributions within their papers.

These metric data can serve to validate a tumor bank's existence and successful function. One can also look more qualitatively at the contents of each scientific publication to define those papers that could be construed to be critical to the advancement of medical science, however, the details of this approach are beyond the scope of this chapter. Finally, it should be noted that the BPC's role is not to just serve the established investigator; a bank should also have the discretionary power to support the young investigator with exciting new scientific ideas.

\section{FUTURE CHALLENGES}

Tumor banking to advance medical genetic research has recently been identified as an international priority (12). It has also changed the role of the pathologist from that of simply a tissue refiner to include a role as a data miner (13). It is important in such an enterprise to establish a long-term commitment to substantial resources and secure funding (12); however, the substantive challenges to the success of the enterprise in the future are really more based on tissue-, informatics-, or ethics-based questions.

\section{Specific Challenges}

The specific challenges which need to be addressed by any tumor banking operation in the future include: (i) doing more research with less tissue; (ii) addressing biohazards issues in all phases of the banking operation; and (iii) ensuring informed consent and maintenance of patient confidentiality.

\section{More Research with Less Tissue}

In their editorial on "Looking Forward in Pathology" (1), NIH pathologists noted that "the emphasis is, and will continue to be, on getting more and more specific infor- 
mation from less and less material." The BPC's experience with tumor procurement has been that for most pediatric cancers, about $0.5 \mathrm{~g}$ of snap-frozen tumor is procured per case for research, while in adult gynecologic tumors, the average is more likely $1.0 \mathrm{~g}$ of tissue. In either case, neither amount is sufficient to service multiple investigators without careful forethought and planning.

While it is true that better education of surgeons, pathologists, and tumor procurement personnel may improve the yields of the procured tumor for research, ultimately, the amount of tissue made available for research, which would otherwise be discarded once diagnostic needs are met, is limited by institutional review boards (IRBs) (12). This limitation of tissues procured is further magnified by the growth in use of coreneedle biopsies and fine-needle aspirations as diagnostic procedures.

Given these limitations on amounts of tissue, preservation on-site of mRNA by timely snap-freezing of tissues in liquid nitrogen becomes paramount.

The ultimate role of the pathologist as a tumor banker in this setting is that of a tissue refiner. One of the chief complaints of investigators concerning tumor banks is the lack of sizeable specimens received for their research. It is incumbent upon the pathologists-bankers, as they review a research proposal, to educate the investigator both on the inherent limitations of tissue acquisition and on alternate research techniques that utilize less tissue. Fortunately, with the advent and increased usage of nucleic acid amplification techniques and FISH, such alternate techniques are more readily available; moreover, use of microarray technologies, combined with nucleic acid amplification, may allow for screening of thousands of genes (1).

\section{Biohazards in the Workplace}

With the increasing numbers of individuals infected with hepatitis $\mathrm{C}$ or human immunodeficiency virus (HIV) in the U.S. (14), it becomes incumbent upon any banking operation to operate under the mandate of "universal precautions," even though the BPC does not knowingly accept infected tissues as a stated policy. There are four steps in developing a biosafety program (14), which include: (i) identifying governmental and accrediting agency requirements; (ii) identifying site-based risks and biosafety issues; (iii) developing written working guidelines to improve site-based biosafety; and (iv) implementing a training program.

The BPC maintains such a biosafety program in its banking operations and provides yearly universal precautions training to its procuring hospital personnel at cooperative group meetings. Training of the investigator's personnel in universal precautions is a written expectation of the BPC, which must be signed-off on by investigators, before they receive tissues (6).

Biosafety hazards, as related to specimen containers and transport, have led to changes in the rules concerning transport of biologic specimens (14). Information concerning such regulations is available through links at the Occupational Safety and Heath Administration Web site (http://www.osha.gov) (14).

\section{Informed Consent and Patient Confidentiality}

In a recent editorial (12) on tumor banking, it was noted that "Ironically, it is the power of modern genetic analysis that creates the most difficult ethical dilemmas... concern that 'genetic research' may uncover information that is unwanted by the patient, has implications for family members, and could potentially lead to discrimination." 
The keys to successfully addressing these dilemmas are to: (i) precisely define the meanings of a genetic test and genetic research (15); (ii) define and implement model consent forms that address these definitions (16) and meet IRB expectations; and (iii) maintain the confidentiality of the flow of information from the institution procuring the specimens to the researcher testing them (10). The tumor bank could effectively serve in the latter role by coding or de-linking tissues provided to researchers, managing updates of records, and handling requests for further follow-up data, while maintaining the fiduciary responsibility of protecting patient anonymity (10).

With the evolution of final federal privacy rules concerning "Standards for Privacy of Individually Identifiable Health Information," tumor banks may need to take additional steps to successfully balance the demand for protection of patient privacy with the need to advance medical research. It is clear, from investigator surveys, that researchers are demanding more associated clinical data with their specimens. Improving the informed consent process to allow for acquisition of such data is the key to advancing such translational research.

\section{REFERENCES}

1. Abati, A. and Liotta, L. A. (1996) Looking forward in diagnostic pathology, the molecular superhighway. Cancer 78, 1-3.

2. Grizzle, W. E. and Sexton, K. C. (1999) Development of a facility to supply human tissues to aid in medical research, in Molecular Pathology of Early Cancer, Ch. 24. (Srivastava, S., Henson, D. E., and Gazdar, A., eds.), IOS Press, Washington, D.C., pp. 371-383.

3. Naber, S. P., Smith, L. L., and Wolfe, H. J. (1992) Role of the frozen tissue bank in molecular pathology. Diagn. Mol. Pathol. 1, 73-79.

4. Naber, S. P. (1996) Continuing role of a frozen-tissue bank in molecular pathology. Diagn. Mol. Pathol. 5, 253-259.

5. Farkas, D. H., Kaul, K. L., Wiedbrauk, D. L., and Kiechle, F. L. (1996) Specimen collection and storage for diagnostic molecular pathology investigation. Arch. Pathol. Lab. Med. 120, 591-596.

6. Grizzle, W. E., Aamodt, R., Clausen, K., LiVolsi, V., Pretlow, T. G., and Qualman, S. (1998) Providing human tissues for research: how to establish a program. Arch. Pathol. Lab. Med. 122, 1065-1076.

7. Camp, R. L., Charette, L. A., and Rim, D. L. (2000) Validation of tissue microarray technology in breast carcinoma. Lab. Invest. 80, 1943-1949.

8. Neville, R. (2000) Children's Research Institute core technologies database architecture guide. Internal document, Children's Hospital, Inc., Columbus, OH, May, 2000 (on file).

9. Report of the Intergroup Specimen Banking Committee, Cancer Therapy Evaluation Program, Division of Cancer Treatment and Diagnosis, National Cancer Institute, May 31, 1999 (on file).

10. Merz, J. F., Saukar, P., Taube, S. E., and LiVolsi, V. (1997) Use of human tissues in research: clarifying clinician and research roles and information flows. J. Invest. Med. 45, 252-257.

11. Jewell, S. D., McCart, L. M., Williams, N., et al. (2002) Analysis of the molecular quality of human tissues: an experience from the Cooperative Human Tissue Network (CHTN). Am. J. Clin. Pathol. 118, 733-741.

12. Balleine, R. L., Humphrey, K. E., and Clarke, C. L. (2001) Tumor banks: providing human tissue for cancer research. Med. J. Aust. 175, 293-294.

13. Becich, M. J. (2000) The role of the pathologist as tissue refiner and data miner: the impact of functional genomics on the modern pathology laboratory and the critical roles of pathology informatics and bioinformatics. Mol. Diagn. 5, 287-299. 
14. Grizzle, W. E. and Fredenburgh, J. (2001) Avoiding biohazards in medical veterinary and research laboratories. Biotech. Histochem. 76, 183-206.

15. Grizzle, W., Grody, W., Noll, W., et al. (1999) Recommended policies for cases of human tissue in research, education, and quality control. Arch. Pathol. Lab. Med. 123, 296-300.

16. Taube, S. E., Barr, P., LiVolsi, V., and Pinn, V. W. (1998) Ensuring the availability of specimens for research. Breast J. 4, 391-395. 